\title{
Interhemispheric transfer of colour and shape information in the presence and absence of the corpus callosum
}

\author{
Bettina Forster*, Michael C. Corballis \\ Research Centre for Cognitive Neuroscience, Department of Psychology, University of Auckland, Private Bag 92019, Auckland, New Zealand
}

Received 13 May 1998; received in revised form 9 March 1999; accepted 10 March 1999

\begin{abstract}
Two split-brained subjects, one (L.B.) with full forebrain commissurotomy and one (R.B.) with callosal agenesis, and a group of twenty neurologically intact subjects were tested in three discrimination tasks: a go-no go task, a two-choice task, and a three-choice task. The discriminations were based on colour in Experiment 1, and on shape in Experiment 2. The stimuli were presented in one or other visual field, and the subjects responded with the fingers of one or other hand, allowing the differences in reaction time between crossed and uncrossed responses (CUD) to be calculated. For the normal subjects the CUD tended to diminish with the complexity of the tasks, suggesting that both hemispheres were increasingly involved. Unlike R.B. and the normal controls, who made virtually no errors, L.B. had increasing difficulty as task complexity increased. He was better able to transfer information from the right to the left hemisphere than vice versa, but an analysis of his accuracy under the crossed conditions showed that the amount transferred was always well under one bit. This confirms previous evidence that L.B. has very limited subcortical transfer of either colour or shape. (C) 1999 Elsevier Science Ltd. All rights reserved.
\end{abstract}

\section{Introduction}

Poffenberger [39] proposed that interhemispheric transmission time (IHTT) in the human brain might be measured by subtracting simple reaction time (RT) under uncrossed conditions, where stimulus processing and response initiation take place in the same hemisphere, from RT under crossed conditions, where stimulus processing and response initiation are processed in opposite hemispheres. This crosseduncrossed difference (CUD) is taken to be a direct estimate of IHTT. Numerous studies have employed Poffenberger's paradigm in simple RT tasks (see $[4,8]$ for reviews), and in people with intact corpus callosums the CUD is generally of the order of 2-6 ms, although reported values have ranged from -0.5 to $15.1 \mathrm{~ms}[8]$.

\footnotetext{
* Corresponding author. Present address: Dipartimento di Scienze Neurologiche e della Visione, Universita di Verona, Strada Le Grazie 8, I-37134 Verona, Italy. Fax: +39-045-580881.

E-mail address: bettinaf@borgoroma.univr.it (B. Forster)
}

Poffenberger reasoned further that this technique would be valid only under conditions in which cognitive and response demands are minimised, as in simple RT. Relative to the large number of studies using simple RT, there have been few attempts to measure CUD in more complex information-processing tasks. One such task, involving only a slight increase in complexity, is simple detection, which differs only from simple RT in that blank catch trials are randomly interspersed with target trials. This ensures that the stimulus is processed before a response is initiated. A review of five experiments on simple detection suggested that this slight increase in task complexity creates longer RTs and greater variability in CUD, which ranged from 5 to $28.5 \mathrm{~ms}$ in the reviewed studies $[4,8]$. In a more recent study, however, Brysbaert [9] found a constant CUD of $2.4 \mathrm{~ms}$ in simple detection experiments with varying numbers of catch trials.

Another level of complexity is added with go-no go tasks, in which the stimulus varies but the subject must respond to only one of two or more alternatives. This means that stimulus discrimination must take place before a response is initiated. Umiltá et al. [48] con- 
ducted three go-no go experiments with increasing stimulus complexity (one-, two-, and three-letter displays). They reported increasing RTs with increasing display complexity, but decreasing CUDs of 2.6, 2.4 and $0.1 \mathrm{~ms}$.

A further information-processing component is added in two-choice tasks, where the subject has to decide which of two responses to make to each of two different stimuli or classes of stimuli. In this case, the CUDs are typically longer than in SRT tasks, with values ranging from $13 \mathrm{~ms}$ [32] to $25 \mathrm{~ms}$ [4] being reported. Response requirements differ somewhat across studies (e.g., one or other hand, one or other finger, one or other direction of movement, etc) which might be mediated by different cerebral mechanisms, allowing only cautious conclusions on the effects of task complexity. Furthermore, in many studies the advantage of uncrossed over crossed conditions has been attributed to spatial compatibility rather than interhemispheric transfer.

These experiments give somewhat conflicting information on the effect of complexity on the CUD. One of the aims of the present experiments, therefore, was to provide further data on this issue. Normal subjects performed three tasks of increasing complexity: a gono go task, a two-choice discrimination, and a threechoice discrimination. The responses were contingent on the colour of the stimuli in Experiment 1, and on the shape of the stimuli in Experiment 2.

A second aim of the study was to examine the performance, on the same three tasks, of two further subjects who lack the corpus callosum. One is a young man (R.B.) with agenesis of the corpus callosum, and the other an older man (L.B.) with surgical section of the forebrain commissures, including the anterior and hippocampal commissures as well as the corpus callosum. In commissurotomised subjects, CUDs based on simple RT vary between about 29 and $96 \mathrm{~ms}$, indicating that transfer via extracallosal pathways is substantially longer than that via the corpus callosum $[10,13,21,40]$. People with agenesis of the corpus callosum have also been found to have CUDs that are prolonged relative to those in normals, although typically shorter than those with surgical section of the commissures; in various studies, they have ranged between 9.5 and $32 \mathrm{~ms}[1,13,21,24,28,37,45]$. They may also show other subtle deficits in interhemispheric interaction besides this increase in CUD $[15,31,33,36]$, although they do not show the now classic patterns of disconnection evident in callosotomised or commissurotomized subjects $[5,25,30]$.

By adding to the complexity of the task, our experiments bear on further issues concerning the nature of interhemispheric transfer in the absence of the corpus callosum. Studies of the CUD using simple RT have shown that people lacking the corpus callosum and other forebrain commissures can transfer simple information between hemispheres, albeit more slowly than in normals, and there has been some discussion as to whether the information transferred is stimulus information or response information. Evidence from neurologically intact subjects suggests that the CUD is largely unaffected by variations in luminance or eccentricity $[4,8,10,21]$ suggesting that the information transferred is insensitive to visual parameters, and is therefore more likely to be motor than sensory. Further, the CUD in both the commissurotomized subject L.B. and the acallosal subject R.B. has been found to be largely unaffected by variations in luminance, including a condition of equiluminance $[1,21]$, again suggesting that the information transferred is motor rather than sensory. A stronger test of the nature of the information transferred is provided in the present experiments, since the responses are contingent upon the colour and shape of the stimuli. At least in the case of L.B., and other subjects with section of the corpus callosum and other forebrain commissures, there is evidence that information about colour and shape does not transfer interhemispherically, or does so very poorly, and the question addressed by the present experiments is whether L.B. can perform accurately on discrimination tasks under crossed conditions.

Previous evidence from two-choice RT experiments does suggest, however, that L.B. can transfer twochoice response information interhemispherically with reasonable accuracy. Although he is typically no better than chance in making binary same-different judgments of colour or form across the visual fields [26], implying that these visual attributes do not transfer, he is reasonably accurate at making binary decisions about stimuli presented wholly within a visual field, as for example in studies of mental rotation [16]. It is likely in this case that the transfer is of response information rather than stimulus information. It has also been suggested that interhemispheric transfer in L.B. may be limited to binary information, which may conceivably be accomplished by rapid external cross-cueing; for example a binary decision might be signalled interhemispherically by raising or lowering the tongue [11]. Consequently, the three-choice discrimination task is of special interest, since accurate performance would require transfer of more than binary information-or, in information-theory terms, more than one binary digit, or 'bit', of information. The present experiments allowed us to measure from L.B.'s performance on the discrimination tasks just how many bits of information were transferred from stimulus to response (see [22] for computational details). Under crossed conditions, this provided a quantitative estimate of how much information was transferred interhemispherically. 


\section{Experiment 1: colour}

In this experiment, the subjects made responses contingent on the colour of the stimuli. Previous evidence suggests that the transfer of colour information is largely abolished following forebrain commissurotomy $[12,26,40,44]$, although it has been suggested that subcortical transfer may enable a crude distinction between short and long wavelengths of light [46]. We have previously shown that L.B.'s CUD was unaltered in a simple RT task when the stimuli were equiluminant with the background, and therefore defined by colour only [21]. This could be taken to mean either that the CUD reflects the transfer of response information rather than stimulus information, or else that it reflects ipsilateral response initiation under the crossed conditions [40].

Evidence as to whether subjects with agenesis of the corpus callosum can transfer colour information interhemispherically is somewhat mixed. Sauerwein and Lassonde [42] reported that acallosal subjects were able to cross integrate tachistoscopically presented colour and shape information although their performance was much slower than that in normal controls. However, Karnath et al. [27] tested an acallosal subject who could not make same-different judgements on the bases of colour or shape for bilaterally presented stimuli, although he could name colours presented unilaterally. As was the case with L.B., our earlier study showed that the acallosal subject R.B.'s CUD was unaltered by equiluminance.

\subsection{Method}

\subsubsection{Subjects}

There were 20 normal subjects, 10 women and 10 men, who participated on a voluntary basis. Their ages ranged from 21 to 29 years with an average of 24 years. Each subject performed all three tasks.

The acallosal subject was R.B., a 20-year old lefthanded man. An MRI examination in 1988, when he was 12 years old, showed that he lacked the corpus callosum, and has a dilatation of the posterior portions of the lateral ventricles (see [21] for MRI scans). Neurological examination was performed since R.B. complained about headaches leading to a suspicion that he might have inherited a form of optic subatrophy [1]. There has been no reason for any further neurological testing. Standard neurological examin-

\footnotetext{
${ }^{1}$ In the case of L.B., in particular, we felt it to be important to initiate each trial, especially since he appeared drowsy, perhaps as a result of some recent left-hemisphere pathology. We also wanted to ensure that trials were not initiated while he was having a (rare) petit mal seizure.
}

ations have revealed no clear manifestation of interhemispheric disconnection [1], except for a lengthened CUD and some subtle sign of defective visual communication between the hemispheres [13,15,20,21].

The commissurotomized subject was L.B., a 45-yearold right-handed man who underwent section of the corpus callosum and the anterior commissure and the hippocampal commissure in 1963. Magnetic resonance imaging (MRI) confirmed complete section of the corpus callosum [6]. Details of the case history and neurological status are available elsewhere [7]. At the time of testing L.B. showed slightly slurred speech and an uncharacteristic listlessness, possibly indicating a recently acquired left-hemispheric dysfunction. We have no reason to suspect that this might have impaired interhemispheric transfer.

\subsubsection{Stimuli and apparatus}

The stimuli comprised filled circular disks, $0.86^{\circ}$ in visual angle, flashed $2.5^{\circ}$ either to the left or right of the central fixation cross. The stimuli were either red, green or blue. Each colour was presented at two different luminance levels (red $=10.07$ and $6.24 \mathrm{~cd} / \mathrm{m}^{2}$, green $=10.04$ and $6.04 \mathrm{~cd} / \mathrm{m}^{2}$ and blue $=9.19$ and 6.09 $\mathrm{cd} / \mathrm{m}^{2}$ ). Since equiluminance between colours could be achieved only approximately, variations in luminance were introduced to prevent subjects from using luminance as the discriminative cue. The stimuli were viewed from a distance of $57 \mathrm{~cm}$ from the screen, so that one $1 \mathrm{~cm}$ on the screen corresponded to $1^{\circ}$ of visual angle. A chin rest was used to minimise head movements. Responses were made on a keyboard placed at the subject's midline. Stimuli were presented on a fast-fade videographics adaptor (VGA) screen. The experiments were programmed with the software package Micro Experimental Laboratory (MEL) [43].

\subsubsection{Procedure}

Each task comprised two practice blocks and four blocks of experimental trials. At the beginning of each block the subject was instructed which hand to use. Stickers corresponding to each colour were placed on the keys selected for response. At the beginning of each experiment subjects were instructed to always fixate on the centre cross and press the key that showed the same colour as the stimulus as fast as possible. Subjects had no difficulty memorising which key stood for which colour, and did not have to check visually. In the case of a false response a short high-pitched pulse was sounded. The normal subjects initiated each trial by pressing the space bar with the non-responding hand, while for R.B. and L.B. the experimenter initiated each trial by pressing either the ' $=$ ' or the ' 1 ' key. ${ }^{1} 500 \mathrm{~ms}$ after a trial was initiated, a stimulus would be presented on the screen for $133 \mathrm{~ms}$. Any re- 
Table 1

Means of median RTs (in ms) of the normal subjects and median RTs (in ms) of R.B. and L.B. for each hand-visual field combination, and corresponding CUDs, in Experiment 1 (proportions correct shown in brackets)

\begin{tabular}{|c|c|c|c|c|c|}
\hline Task & Left hand-LVF & Left hand-RVF & Right hand-LVF & Right hand-RVF & CUD \\
\hline \multicolumn{6}{|c|}{ Normal subjects } \\
\hline Go-no go & $412(0.97)$ & $414(0.97)$ & $412(0.99)$ & $402(0.98)$ & 6 \\
\hline Two-choice & $475(0.97)$ & $469(0.98)$ & $452(0.97)$ & $449(0.96)$ & -1 \\
\hline Three-choice & $558(0.98)$ & $551(0.97)$ & $551(0.95)$ & $550(0.97)$ & -3 \\
\hline \multicolumn{6}{|c|}{ Acallosal subject R.B. } \\
\hline Go-no go & $398(1.00)$ & $396(1.00)$ & $401(1.00)$ & $413(1.00)$ & -7 \\
\hline Two-choice & $485(0.98)$ & $521(0.98)$ & $514(1.00)$ & $552(0.93)$ & -1 \\
\hline Three-choice & $477(1.00)$ & $539(0.97)$ & $561(0.99)$ & $519(0.96)$ & 51 \\
\hline \multicolumn{6}{|c|}{ Commissurotomised subject L.B. } \\
\hline Go-no go & $483(1.00)$ & $576(1.00)$ & $685(1.00)$ & $632(1.00)$ & 73 \\
\hline Two-choice & $744(0.93)$ & $949(0.73)$ & $903(0.84)$ & $845(0.96)$ & 132 \\
\hline Three-choice & $784(1.00)$ & $1146(0.54)$ & $1042(0.75)$ & $938(0.93)$ & 233 \\
\hline
\end{tabular}

sponses occurring while the stimulus was on the screen were treated as anticipations and were not recorded.

On each task, the subjects were first given two blocks of eight practice trials, one with each hand. They were then given four blocks of experimental trials. Half of the normal subjects performed these blocks with the left hand, right hand, right hand, and left hand respectively, while for the other half this ordering was reversed. For both R.B. and L.B., the order was left, right, right, left.

The specific tasks were as follows:

2.1.3.1. Go-no go task. Each subject was instructed to press the key with the red sticker on the keyboard only when a red disk flashes up on the screen. On half of the trials the disc was red, and on the other half it was green. Thus no response or an inhibition of response was required for half of the trials. Each block of experimental trials contained 56 trials, 28 each visual field, so there was a total of 224 trials over the four blocks. On half of the trials in each field the disk was red and on the other half it was green. These conditions were presented in random order. In no-response trials there was a delay of $3000 \mathrm{~ms}$ before the instruction for the initiation of the next trial appeared at the bottom of the screen.

2.1.3.2. Two-choice task. The procedure was the same as in the previous task, except that the subjects were required to press the 'red' or the 'green' key according to the colour they saw by using the forefinger, and middle finger of the same hand. The B key served as the 'red' key, and the $\mathrm{N}$ key as the 'green' key. Again, the experimental blocks comprised 56 trials per block, making a total of 224 trials.

2.1.3.3. Three-choice task. In this experiment subjects had to indicate whether a red, green or blue disk had been flashed on the screen by pressing the $\mathrm{B}, \mathrm{N}$, or $\mathrm{M}$ keys, respectively, which were marked with the appropriate colours. They were instructed to use the forefinger, middle finger, and ring finger of the same hand. The four experimental blocks contained 72 trials each, making a total of 288 trials. For reasons explained below, L.B. was given an extra session of two practice and two (instead of four) experimental blocks.

\subsection{Results}

\subsubsection{Normal subjects}

Table 1 summarises the RTs and accuracy of performance under each hand-field combination for each task. Accuracy was too high to warrant further analysis.

For correct responses, median RTs for each hand and visual field were calculated, and subjected to analyses of variance, with hand and visual field as withinsubject factors, and gender as a between-subject factor. In no cases were there any significant effects or interactions associated with gender. For the two-choice and three-choice tasks, colour was an additional withinsubject factor. The values shown in Table 1 are the means of the medians, and the resulting CUDs.

2.2.1.1. Go-no go task. The false-alarm rate was $1.8 \%$. The analysis of variance revealed no significant main effects of hand or visual field. The interaction between the two, reflecting a CUD of $6 \mathrm{~ms}$, reached significance on a one-tailed test $(F(1,18)=4.23$, one-tailed $P<0.05)$, despite a range of individual values $-20-56$ ms.

2.2.1.2. Two-choice task. The main effect of hand was significant $(F(1,18)=11.66, P<0.005)$ with the right hand being on average $27 \mathrm{~ms}$ faster than the left. Neither the main effect of visual field nor the interaction between hand and visual field interaction $(F(1$, $18)=0.06$, n.s.) were significant. The mean CUD was 
slightly negative $(-1 \mathrm{~ms})$ with individual values ranging from -30 to $39 \mathrm{~ms}$.

There were significant interactions between hand and colour $(F(1,18)=5.34, P<0.05)$ and between field and colour $(F(1,18)=72.27, \quad P<0.0001)$, suggesting a colour-contingent hemispheric asymmetry. Tests of simple effects showed a right-hand $(t(35)=2.75, \quad P<0.005)$ and a RVF $(t(31)=4.06$, $P<0.0001)$ advantage in responding to green, and a LVF $(t(31)=4.26, P<0.0001)$ advantage in responding to red. A right-hand advantage in responding red was not significant $(t(35)=0.77$, n.s. $)$. These effects might be attributed to stimulus-response compatibility, since the 'red' key was to the left and the 'green' key to the right.

2.2.1.3. Three-choice task. There were no significant effects of hand or of visual field, nor any significant interactions involving hand. The median RT for the uncrossed condition (554 ms) is again very slightly slower than of the crossed condition (551 ms), yielding a mean CUD of $-3 \mathrm{~ms}$, but the interaction between hand and visual field did not approach significance. The individual CUDs between -31 and $25 \mathrm{~ms}$.

There was again a significant field-by-colour interaction $(F(2,36)=22.89, P<0.0001)$ which was attributable to faster responses to blue than to green or red in the $\operatorname{RVF}(t(50)=3.78, P<0.0001)$, and faster to red than green and to blue in the $\operatorname{LVF}(t(50)=3.69$, $P<0.0001)$. Again, these effects are attributable to stimulus-response compatibility, since the response keys, from left to right, were 'red', 'green', and 'blue'.

2.2.1.4. Discussion. The median RT increased with increasing task complexity, but the mean CUD decreased, if anything, and was virtually zero (and actually slightly negative) for the two-choice and threechoice tasks. The mean CUD of $6 \mathrm{~ms}$ for the go-no go task is in marked contrast to the prolonged CUD for simple detection tasks reported in the studies reviewed by Bashore [4], but is consistent with the more recent results of Brysbaert [9]. The absence of any RT advantage under the crossed conditions in the two-choice and three-choice tasks supports Poffenberger's [39] suggestion that the use of the CUD as a measure of IHTT applies only to simple RT tasks, and may not be valid for the effects found in these more complex tasks. A possible reason for the reduction in the CUD with increased complexity is that both hemi-

\footnotetext{
${ }^{2}$ Unlike any of the normals, R.B. is left-handed, and L.B. is outside the age range of the normal sample, which was not intended as a control sample. Comparisons therefore should be viewed with caution, although we have no reason to suspect that handedess or age has a marked effect on interhemispheric transfer.
}

spheres are increasingly involved in the task, even under uncrossed conditions. However CUDs showed quite some variability between subjects possibly due to the small amount of trials.

\subsubsection{R.B.}

Table 1 shows R.B.'s median RTs and accuracy under each hand-field combination for each task, along with his CUDs. His accuracy was well within the normal range. ${ }^{2}$ Analyses of variance was carried out on all RTs where he responded correctly, using a single-subject design, so that the results cannot be generalized to acallosal populations.

2.2.2.1. Go-no go task. R.B. responded on $6 \%$ of 'nogo' trials. Neither the main effect of hand $(F(1$, $108)=0.14$, n.s. $)$, nor of visual field $(F(1,108)=0.00$, n.s.), nor the interaction between hand and field $(F(1$, $108)=0.72$, n.s.) were significant. The CUD of $-7 \mathrm{~ms}$ is well within the range of values from the normal subjects.

2.2.2.2. Two-choice task. As Table 1 shows, R.B.'s accuracy was generally high but dropped to $93 \%$ correct in the right hand-RVF condition. Nevertheless a multidimensional chi-square analysis [49] revealed no significant effects of colour $\left(\chi^{2}(1, N=224)=2.74\right.$, n.s. $)$, visual field $\left(\chi^{2}(1, N=224)=2.74\right.$, n.s. $)$ or hand used $\left(\chi^{2}(1, N=224)=0.68\right.$, n.s. $)$ on response selection, nor were any of their interactions significant. R.B.'s overall median RT of 518 ms was longer than in the previous task. His RT was on average $44 \mathrm{~ms}$ shorter to $\mathrm{LVF}$ than to RVF stimuli, $(F(1,210)=4.76$, $P<0.05)$, but the interaction between field and hand was not significant $(F(1,210)=0.67$, n.s. $)$. Neither the main effect of hand $(F(1,210)=2.65$, n.s. $)$ nor that of colour $(F(1,210)=1.92$, n.s. $)$, nor any of their interactions reached significance. The CUD was effectively zero $(-1 \mathrm{~ms})$, which is again within the normal range.

2.2.2.3. Three-choice task. There were no significant main effects or interactions on either RT or response accuracy. The CUD of $52 \mathrm{~ms}$ was beyond the normal range, but was still not significantly different from zero.

2.2.2.4. Discussion. There was little indication from these results that R.B.'s performance differed in any systematic way from that of the normal subjects. Despite some loss of accuracy in the right-hand-RVF condition in two-choice discrimination, his performance remained high under all other conditions, including the three-choice task. The number of trials was perhaps too small to yield accurate measures of the CUD, but the values obtained in the go-no go and two-choice tasks are if anything shorter than expected, 
given previous evidence for a somewhat prolonged CUD as estimated from simple RT [1,13,21,47]. The prolonged CUD of $51 \mathrm{~ms}$ in the three-choice task is tantalising, but was not significantly different from zero, perhaps due to the small sample size and high variability.

\subsubsection{L.B.}

Table 1 shows L.B.'s median RTs and accuracy under each hand-field combination for each task. His CUDs are also shown. Analysis of variance is again based on a single-subject design, so the results cannot be generalised to the population of commissurotomised subjects.

2.2.3.1. Go-no go task. L.B. responded correctly on every 'go' trial but he also responded on $13 \%$ of 'nogo' trials. Analysis of variance of his RTs revealed a main effect of hand $(F(1,108)=29.93, P<0.001)$. His right-hand responses were on average $149 \mathrm{~ms}$ slower than his left-hand responses. A similar effect was observed in an earlier study on simple RT [21], and may be related to other signs of left-hemisphere pathology that L.B. exhibited at the time of testing - these included some slurring of speech and a general sluggishness. There was no significant difference in RT between visual fields, but there was a significant interaction between visual field and hand $(F(1,108)=4.63$, $P<0.05)$, reflecting a CUD of $73 \mathrm{~ms}$. This is beyond the normal range, but within the range of values, based on simple RT, in a previous report [21].

2.2.3.2. Two-choice task. Since L.B.'s accuracy dropped to $73 \%$ in the RVF-left hand condition and to $84 \%$ in the LVF-right hand condition, multidimensional chisquare analysis were first carried out to determine the extent to which response selection depended on stimulus manipulations. Response selection depended significantly on the interaction between hand and field $\left(\chi^{2}(1\right.$, $N=224)=12.47, P<0.001)$, reflecting the loss of accuracy in crossed relative to uncrossed conditions (Table 1). There was also a significant interaction between colour and visual field $\left(\chi^{2}(1, N=224)=7.54\right.$, $P<0.01$ ), reflecting a LVF advantage for red and a RVF advantage for green. There was a similar interaction between colour and hand $\left(\chi^{2}(1\right.$, $N=224)=3.84, P<0.05)$ a right-hand advantage for red and a left-hand advantage for green. Again, these effects are probably due to stimulus-response compatibility. The interaction between hand and field was not significantly influenced by colour.

The information transmitted in each hand-by-field combination was calculated by subtracting the uncertainty about which response will occur for each stimulus presented from the amount of information that is contained in the uncertainty about the response [22].
Table 2

Information transferred (in bits) based on L.B.'s response allocation for each combination of hand and visual field in Experiment 1

\begin{tabular}{|c|c|c|c|c|c|c|c|}
\hline \multicolumn{3}{|c|}{ Two-choice task } & \multicolumn{3}{|c|}{$\begin{array}{l}\text { Three-choice task } \\
\text { (first session) }\end{array}$} & \multicolumn{2}{|c|}{$\begin{array}{l}\text { Three-choice task } \\
\text { (second session) }\end{array}$} \\
\hline \multirow[b]{2}{*}{ Field } & \multicolumn{2}{|c|}{ Hand } & \multirow[b]{2}{*}{ Field } & \multicolumn{2}{|c|}{ Hand } & \multirow[b]{2}{*}{ Field } & Hand \\
\hline & Left & Right & & Left & Right & & Left Right \\
\hline Right & 0.17 & 0.77 & Right & 0.24 & 1.06 & Right & $0.44 \quad 1.58$ \\
\hline Left & 0.64 & 0.41 & Left & 1.58 & 0.69 & Left & $1.16 \quad 0.61$ \\
\hline
\end{tabular}

Information is measured in binary digits ('bits'), such that the choice of one stimulus from two equiprobable stimuli represents one bit. L.B. transferred only 0.17 bits in left-hand responses to RVF stimuli, and 0.41 bits in right-hand responses to LVF stimuli (corresponding to $73 \%$ and $84 \%$ correct responses, respectively) (see Table 2).

Analysis of L.B.'s RTs for correct responses revealed no main effect of hand, contrary to the results on the previous task. However, there was a marked effect of visual field, $(F(1,186)=4.01, P<0.05)$, with mean RT to LVF stimuli being $115 \mathrm{~ms}$ shorter than mean RT to RVF stimuli. The interaction between hand and visual field in the predicted direction, and significant according to a one-tailed test $(F(1,186)=3.12$, one-tailed $P<0.05)$. The median RT for crossed responses of $926 \mathrm{~ms}$ is clearly lengthened in comparison to the RT of $795 \mathrm{~ms}$ for uncrossed responses, yielding a CUD of $132 \mathrm{~ms}$. There were no significant effects or interactions associated with colour.

2.2.3.3. Three-choice task: session 1. Multidimensional chi-square analysis confirmed the large difference in accuracy between crossed and uncrossed conditions $\left(\chi^{2}\right.$ $(1, N=288)=46.91, P<0.001)$. The same pattern of interactions between colour and visual field $\left(\chi^{2}(2\right.$, $N=288)=6.56, P<0.05)$ and between colour and hand $\left(\chi^{2}(2, N=288)=7.20, P<0.05\right)$ as in the twochoice task was found, namely, a LVF and left-hand advantage for red, and a RVF and right-hand advantage for green. L.B.'s response accuracy for blue stimuli was always between that for red and green. These effects were probably influenced by stimulus-response compatibility, although it was the 'blue' key, not the 'green' key, that was to the right.

Further, the accuracy levels of 54 and $75 \%$ under the crossed conditions correspond to the transmission of 0.24 and 0.69 bits (in contrast to 1.58 bits required for correct performance), respectively (Table 2), so that information transmission remains at well under one bit. Because of this low level of accuracy, RTs were not subjected to analysis of variance. There was, 
however, an interesting difference in RT for incorrect and correct responses in the crossed conditions. The mean of RT medians of incorrect responses was 1072 ms with a maximum RT of $2280 \mathrm{~ms}$. In contrast, median RT for correct responses was $1127 \mathrm{~ms}$, but the maximum RT was $5109 \mathrm{~ms}$, which is reflected in a larger SD of $748 \mathrm{~ms}$ compared to $297 \mathrm{~ms}$ in the uncrossed condition. Limiting the correct responses to the same RTs range as incorrect responses excludes $11 \%$ of correct crossed responses and reduces the SD to $380 \mathrm{~ms}$. These few slow but correct responses lie well outside range of incorrect responses, and may have been due to cross-cueing. Indeed, L.B. was often observed to be staring at the responding hand, suggesting that the hemisphere receiving the stimulus may have directed his gaze to the appropriate finger or key.

2.2.3.4. Three-choice task: session 2. Since L.B. may have been using a cross-cueing strategy involving the directing of gaze to the appropriate finger or key, we retested him with his hands and the keyboard covered. Only two experimental blocks, each of 72 trials were given following the 2 practice blocks. He used his left hand on the first experimental block, and his right hand on the second.

The effect of covering L.B.'s hands and the keyboard was to reduce accuracy still further under the crossed relative to the uncrossed conditions $\left(\chi^{2}\right.$ (1, $N=144)=31.74, P<0.001)$. However this reduction was limited to the RVF-left hand condition, where his accuracy was $36 \%$ correct, which does not differ significantly from chance $\left(\chi^{2}(1, N=24)=0.125\right.$, n.s. $)$. His performance in the LVF-right hand condition was unaltered at $75 \%$ correct, which was significantly above chance $\left(\chi^{2}(1, \quad N=24)=28.13, \quad P<0.0001\right)$. RTs for correct and incorrect responses were similar, with a maximum at $2700 \mathrm{~ms}$, suggesting that cross-cueing was greatly reduced, if not eliminated.

The amount of information transferred from one hemisphere to the other was limited to 0.44 bits in the left hand-RVF and 0.61 bits in the right hand-LVF combination. Again this is less than both the 1.58 bits required for correct performance and the 1 bit that would be expected if only binary information were transferred (Table 2).

Although L.B.'s left-hand responses to RVF stimuli were not above chance in overall accuracy, there was nevertheless a significant contingency between stimuli and responses $\left(\chi^{2}(4, \quad N=108)=18.8, \quad P<0.001\right)$, despite an overall bias towards responding 'blue' $\left(\chi^{2}(2\right.$, $N=144)=7.53, \quad P<0.025)$. Thus L.B. responded 'blue' on 9 of the 12 occasions on which the stimulus was actually green and 'green' on 9 of the 12 occasions on which it was actually red, and he responded 'red' on only four occasions overall. That is, he tended to divide the stimuli into two categories, which he chose to represent as 'blue' for blue and green, and 'green' for red, suggesting that there may have been transfer of at least crude wavelength information.

2.2.3.5. Discussion. L.B.'s results are in marked contrast to R.B.'s in that his CUDs in the go-no go and two-choice tasks were clearly prolonged relative to normal, and in that his accuracy under the crossed conditions was reduced relative to that in uncrossed conditions, especially in the three-choice task. His CUDs also increased systematically with the complexity of the task (see Table 1), but this is probably not an accurate reflection of transfer time, since accuracy also decreased markedly across the three tasks. Rather, both accuracy and CUD probably reflect the increasing difficulty in transferring information interhemispherically as the complexity of that information increased. This confirms previous evidence $[12,26,40,44]$ that L.B. has little ability to transfer colour information interhemispherically, and suggests also a limitation on his ability to transfer response information. Analysis of the amount of information transferred in the crossed conditions revealed a limitation to a level less than one bit.

In the three-choice task, when responding with his left hand to RVF stimuli, L.B. appeared to adopt a cross-cueing strategy of staring at the appropriate finger, and his performance dropped when this strategy was prevented. His accuracy in right-hand responses to LVF stimuli was higher, and was unaffected when cross-cueing was prevented. These results suggest that cross-cueing is more likely to be initiated by the left hemisphere than by the right hemisphere.

Although L.B. was clearly impaired under the crossed conditions in the two-choice and three-choice tasks, the results suggested that there was at least some transfer of colour information, although the amount of information transferred was always less than one bit. In the three-choice task, when responding with his left hand to RVF colours, L.B.'s strong tendency to respond 'blue' to both blue and green, and 'green' to red lends support to Trevarthen and Sperry's [47] conclusion that that there is crude subcortical transfer of wavelength information, allowing some discrimination of short from long wavelengths.

\section{Experiment 2: shape}

Like colour information, shape information does not appear to be transferred following callosotomy $[19,23]$ or full forebrain commissurotomy [26]. More specifically L.B., the commissurotomized subject in this experiment, was unable to compare pairs of patterns, numbers, letters, or colours when presented bi- 
Table 3

Means of median RTs (in ms) of the normal subjects and median RTs (in ms) of R.B. and L.B. for each hand-visual field combination, and corresponding CUDs, in Experiment 2 (proportions correct shown in brackets)

\begin{tabular}{|c|c|c|c|c|c|}
\hline Task & Left hand-LVF & Left hand-RVF & Right hand-LVF & Right hand-RVF & CUD \\
\hline \multicolumn{6}{|c|}{ Normal subjects } \\
\hline Go-no go & $406(1.00)$ & $400(1.00)$ & $420(1.00)$ & $405(1.00)$ & 5 \\
\hline Two-choice & $484(0.97)$ & $470(0.98)$ & $462(0.97)$ & $465(0.98)$ & -9 \\
\hline Three-choice & $569(0.97)$ & $554(0.98)$ & $538(0.97)$ & $541(0.98)$ & -9 \\
\hline \multicolumn{6}{|c|}{ Acallosal subject R.B. } \\
\hline Go-no go & $373(1.00)$ & $364(1.00)$ & $408(1.00)$ & $378(1.00)$ & 11 \\
\hline Two-choice & $471(1.00)$ & $478(0.98)$ & $512(0.96)$ & $479(1.00)$ & 20 \\
\hline Three-choice & $540(1.00)$ & $565(0.99)$ & $566(1.00)$ & $602(1.00)$ & -3 \\
\hline \multicolumn{6}{|c|}{ Commissurotomised subject L.B. } \\
\hline Go-no go & $489(1.00)$ & $553(0.92)$ & $681(1.00)$ & $655(1.00)$ & 45 \\
\hline Two-choice & $740(0.98)$ & $1034(0.75)$ & $864(0.83)$ & $821(0.94)$ & 168 \\
\hline Three-choice & $755(1.00)$ & $919(0.58)$ & $1146(0.81)$ & $995(0.96)$ & 158 \\
\hline
\end{tabular}

laterally, although he could name both stimuli at well above chance, which Johnson [26] attributed to some degree of right-hemispheric (as well as left-hemispheric) control of speech [12]. Corballis and Trudel [18] also found that L.B. failed to score at better than chance in deciding whether four-letter strings straddling the midline comprised words or not, again suggesting a failure to transfer information about shape.

Sauerwein and Lassonde [42] reported that acallosal subjects were able to cross integrate tachistoscopically presented shape information, although as was the case with colour information their performance was much slower than that in normal controls. However, the acallosal subject tested by Karnath et al. [27] could not make same-different judgements on the bases of either colour or shape for bilaterally presented stimuli.

In this experiment, R.B. and L.B. again served as subjects, along with 20 neurologically intact subjects. The tasks were the same as those in Experiment 1, except that the stimuli were simple geometric shapes instead of coloured disks.

\subsection{Method}

\subsubsection{Subjects}

The normal subjects were 10 females and 10 males, who participated on a voluntary basis. Their ages ranged from 21 to 29 years with an average of 25 years. Each subject took part in all three tasks, and none had participated in Experiment 1. The acallosal subject R.B. and the commissurotomized subject L.B. also served.

\subsubsection{Stimuli and procedure}

Stimulus presentation and the general procedure were the same as in Experiment 1. Instead of coloured disks, the stimuli consisted of filled forms, a circle, a triangle and a square. They were equated as far as possible for overall size, with each covering about $1.2 \mathrm{~mm}^{2}$ of white surface area, with a luminance of $64.9 \mathrm{~cd} / \mathrm{m}^{2}$, on a black background.

In the go-no go task, the stimuli were circles and squares, and the subjects were to respond to the circles but not to the squares. In each of the four experimental blocks, there were 48 trials, 24 in each visual field, making a total of 192 trials. The two-choice task was the same, except that subjects were to press one key (N) to circles and another (M) to squares. In the threechoice task, the stimuli were circles, squares, and triangles, and the corresponding keys were $\mathrm{B}, \mathrm{N}$, and $\mathrm{M}$. There were 72 trials in each experimental block, making a total of 288 trials. The keys were indicated by stickers showing the appropriate shape.

\subsection{Results}

\subsubsection{Normal subjects}

Table 3 summarises the RTs and accuracy of performance under each hand-field combination for each task. Accuracy was too high to warrant further analysis, but median RTs for correct responses were again subjects to analysis of variance.

3.2.1.1. Go-no go task. Only $0.8 \%$ of false-alarms were registered. For each subject the median RT for correct responses was calculated for each condition of visual field and hand. The median RTs were then subjected to separate analysis of variance, with visual field and hand as within-subject factors and gender as betweensubject factors. There was a significant gender effect $(F(1,18)=4.63, P<0.05)$ with the men responding on average $100 \mathrm{~ms}$ faster than the women, and a significant main effect of visual field $(F(1,18)=8.71$, $P<0.0001$ ), with faster responses to right-visual-field stimulation by about $10 \mathrm{~ms}$. However, no other main effect or interaction reached significance; there was no indication, for example, that the CUD depended on 
gender. The overall CUD of $5 \mathrm{~ms}$ did not result in a significant interaction between hand and visual field $(F(1,18)=1.69$, n.s. $)$; individual values ranged from -14.5 to $45.5 \mathrm{~ms}$.

3.2.1.2. Two-choice task. The median RTs were again subjected to separate analysis of variance, with each visual field, hand, and shape as within-subject factors and gender as a between-subject factor. Neither the main effect of hand nor that of visual field was significant, nor did the interaction between hand and visual field reach significance $(F(1,18)=2.80$, n.s. $)$ which is reflected in some variability of CUD between subjects ( $-56-42 \mathrm{~ms})$. The mean of the median RTs uncrossed responses $(475 \mathrm{~ms})$ is slower than that for crossed responses $(466 \mathrm{~ms})$, yielding a negative mean CUD ( -9 $\mathrm{ms})$.

There was no significant main effect of shape. However a significant shape-by-field interaction $(F(1,18)=39.63, P<0.0001)$ indicates faster responses to circles in the LVF than in the RVF $(t(36)=3.50$, $P<0.0001)$ and, faster responses to squares in the RVF than in the LVF $(t(36)=5.03, P<0.0001)$. These effects were probably due to stimulus-response compatibility, since the 'circle' key was to the left of the 'square' key. There were no significant effects or interactions associated with gender.

3.2.1.3. Three-choice task. Median RTs were again subjected to analysis of variance. The main effect of visual field was not significant. However, there was a significant main effect of hand $(F(1,18)=5.79, P<0.05)$, with right hand responses being about $22 \mathrm{~ms}$ faster than left hand responses. Although CUDs ranged between $-44 \mathrm{~ms}$ and $11 \mathrm{~ms}$ with a negative mean CUD of $-9 \mathrm{~ms}$ there was a significant interaction between hand and visual field $(F(1,18)=6.43$, $P<0.025)$.

There was a significant difference in RTs between the three forms $(F(2,36)=7.50, P<0.025)$ with RTs being faster for squares than for circles and triangles. There was a significant shape-by-field interaction $(F(2,36)=16.16, \quad P<0.0001)$, and tests of simple effects revealed significantly faster responses to circles in the LVF than in the RVF $(t(52)=2.98, P<0.001)$, and faster responses to triangles in the RVF than in the $\operatorname{LVF}(t(52)=3.34, P<0.0001)$. The mean RT to squares was fastest in the RVF and lay between those to circles and triangles in the LVF. These effects are probably attributable in part to stimulus-response compatibility, since the key for circles was on the left, for triangles on the right, and for squares in between, and in part to faster responding with the forefinger than with the other two fingers. Again, there were no effects or interactions associated with gender.
3.2.1.4. Discussion. With increasing task complexity the median RT increased, but the CUD decreased from 5 $\mathrm{ms}$ in the go-no go task to $-9 \mathrm{~ms}$ in the two-choice and three-choice tasks (Table 3). This essentially confirms the results of Experiment 1, which showed a similar decrease in CUD with increasing task complexity, although in this experiment the CUD was actually significantly negative in the case of the three-choice task. A possible explanation for the negative CUD is that performance is more efficient with shared processing, with stimulus discrimination carried out in one hemisphere and response selection in the other. This means that there might have been some degree of parallel (or overlapping) processing under the crossed conditions [2]. However, there is also quite some variability in the range of CUD between subjects which challenges this overall conclusion.

In all three task an overall RVF advantage was apparent, perhaps reflecting a left hemispheric advantage for response selection [41].

\subsubsection{R.B.}

As shown in Table 3, R.B.'s accuracy on all tasks was very high. Subsequent analysis were restricted to $\mathrm{RTs}$ for correct responses.

3.2.2.1. Go-no go task. No false-alarm was registered in any of the 'no-go' trials. There was no significant effect of hand $(F(1,92)=0.60$, n.s.) or visual field $(F(1,92)=1.01$, n.s. $)$, nor was the interaction between them significant $(F(1,92)=1.40$, n.s. $)$. The median RTs for uncrossed responses $(376 \mathrm{~ms})$ was nevertheless faster than for crossed responses $(386 \mathrm{~ms})$, yielding a CUD of $10 \mathrm{~ms}$.

3.2.2.2. Two-choice task. Neither the main effect of hand $(F(1,81)=0.02$, n.s. $)$ nor that of visual field $(F(1$, $81)=0.47$, n.s.) was significant, but the interaction between the two, which was in the expected direction, reached significance on a one-tailed test $(F(1$, $81)=2.98$, one-tailed $P<0.05$ ). The CUD based on median RTs was $20 \mathrm{~ms}$.

There was no difference between RTs for the two forms $(F(1,81)=0.02$, n.s. $)$, but a significant shape-byfield interaction $(F(1,81)=6.56, P<0.05)$ is attributable to faster responses to squares in the RVF, although this did not reach significance $(t(78)=1.64$, n.s.), and faster responses to circles in the LVF, which reached significance on a one-tailed test $(t(70)=1.93$, one-tailed $P<0.05$ ). These effects are possibly due to stimulus-response compatibility, since the response key for 'circle' was to the left of that for 'square'.

3.2.2.3. Three-choice task. There was a significant main effect of hand $(F(1,275)=4.26, P<0.05)$ with lefthand responses being $9 \mathrm{~ms}$ faster than right-hand re- 
sponses, and of visual field $(F(1,275)=6.53$, $P<0.001$ ), with responses to LVF stimuli being 46 ms faster than responses to RVF stimuli. However, the interaction was not significant $(F(1,275)=0.28$, n.s. $)$. The CUD was $-3 \mathrm{~ms}$.

3.2.2.4. Discussion. It is clear that R.B. had little difficulty with any of the tasks. His accuracy, RTs, and CUDs were all within the normal range. As in Experiment 1, however, his CUDs showed a variable pattern, increasing from the go-no go $(11 \mathrm{~ms})$ to the twochoice task $(20 \mathrm{~ms})$, but decreased to virtually zero on the three-choice task (Table 3). This pattern is the opposite of that observed in Experiment 1, where the CUD was very small in the no-go and two-choice tasks, but increased to $51 \mathrm{~ms}$ in the three-choice task.

\subsubsection{L.B.}

3.2.3.1. Go-no go task. Four percent of false-alarms were registered. L.B. responded $100 \%$ accurately in three of the four hand-field combinations. In the RVFleft hand condition his accuracy dropped to $92 \%$. Multidimensional chi-square analysis showed that response selection depended significantly only on stimulus manipulations $\quad\left(\chi^{2}(1, \quad N=192)=168.82\right.$, $P<0.001)$, but not on hand or field.

Analysis of variance of RTs for correct responses showed that right-hand responses were on average 134 $\mathrm{ms}$ slower than left-hand responses $(F(1,90)=20.15$, $P<0.0001)$. This striking difference was also apparent in Experiment 1, as well as other recent experiments $[13,20]$. There was no significant difference in RTs between visual fields, but a significant interaction between hand and visual field $(F(1,90)=6.01$, $P<0.05)$ reflects shorter RTs to uncrossed than crossed conditions. The CUD of $45 \mathrm{~ms}$ lies just beyond the normal range.

3.2.3.2. Two-choice task. L.B.'s accuracy dropped to $94 \%$ in the RVF-right hand condition, $83 \%$ in the LVF-right hand condition, and $75 \%$ in the RVF-left hand condition (Table 3). Multidimensional chi-square analysis showed that L.B.'s response selection depended significantly on the triple interaction between hand, field, and shape $\left(\chi^{2}(1, \quad N=192)=6.58\right.$, $P<0.025)$; in the crossed hand-by-field combinations he was less accurate with his left hand when the presented shape was a circle and less accurate with his right hand when the presented shape was a square. However, the hand-by-field interaction itself was not significant $\left(\chi^{2}(1, N=192)=0.07\right.$, n.s. $)$.

Analysis of the information transferred from one hemisphere to the other [22] revealed that only 0.21 bits in the left hand-RVF and 0.47 bits in the right hand-LVF combination were transferred. In contrast 1
Table 4

Information transferred (in bits) based on L.B.'s response allocation for each combination of hand and visual field in Experiment 2

\begin{tabular}{|c|c|c|c|c|c|}
\hline \multicolumn{3}{|c|}{ Two-choice task } & \multicolumn{3}{|c|}{ Three-choice task } \\
\hline \multirow[b]{2}{*}{ Field } & \multicolumn{2}{|c|}{ Hand } & \multirow[b]{2}{*}{ Field } & \multicolumn{2}{|c|}{ Hand } \\
\hline & Left & Right & & Left & Right \\
\hline Right & 0.21 & 0.83 & Right & 0.27 & 1.16 \\
\hline Left & 0.88 & 0.47 & Left & 1.33 & 0.95 \\
\hline
\end{tabular}

bit of information is required for binary transfer of information and correct performance in the two choicetask (Table 4).

Analysis of L.B.'s RTs showed no significant main effect of hand, contrary to the left-hand advantage evident in the previous task. However, there was a significant visual field effect $(F(1,160)=4.58, P<0.05)$ with RVF responses being $109 \mathrm{~ms}$ slower than LVF responses. The interaction between hand and visual field was in the predicted direction, and was significant on a one-tailed test $(F(1,160)=3.58$, one-tailed $P<0.05)$. The CUD of $168 \mathrm{~ms}$ is clearly beyond the normal range.

3.2.3.3. Three-choice task. L.B.'s response accuracy in the crossed conditions dropped even further, to $58 \%$ in the left hand-RVF condition and $81 \%$ in the right hand-LVF condition. Analysis was therefore restricted to response accuracy, using multidimensional chisquare analysis. There was a significant interaction between shape and visual field $\left(\chi^{2}(4, N=288)=11.84\right.$, $P<0.025)$, reflecting lower accuracy in the LVF when the stimuli were squares than triangles and circles, and lower accuracy in the RVF when the stimuli were circles or squares than triangles. This effect might be explained by stimulus-response compatibility, since the highest accuracy was for left-key responses (circles) in the LVF and right-key responses (triangles) in the RVF, with middle-key responses (squares) in between. The three forms also affected the hand-by-field interaction in a similar manner $\left(\chi^{2}(4, N=288)=46.88\right.$, $P<0.001)$, in that L.B. showed lower accuracy in the crossed combinations for squares than triangles and circles in the right hand-LVF combination, and for circles than squares and triangles in the left hand-RVF combination. Although L.B.'s accuracy dropped to 58 and $81 \%$ in the left hand-RVF and right hand-LVF conditions, respectively, his response allocation was still contingent on shape $\left(\chi^{2}(4, \quad N=72)=27.15\right.$, $P<0.001$ and $\chi^{2}(4, N=72)=84.4, P<0.001$, respectively). However, in the left hand-RVF condition he responded 'triangle' on $56 \%$ of the trials, perhaps reflecting a bias to respond with the forefinger. An 
informational analysis showed that in the left handRVF combination 0.27 bits and in the right hand-LVF combination 0.95 bits were transferred (Table 4).

L.B.s accuracy was considered too low to warrant statistical analysis of his RTs, but the CUD as computed from median RTs to correct responses was 158 ms.

3.2.3.4. Discussion. In contrast to R.B. L.B.'s accuracy was increasingly impaired with increasing task complexity. Further, his CUD increased from $45 \mathrm{~ms}$ in the go-no go task to $169 \mathrm{~ms}$ in the two-choice task. Since stimulus information is the same in both tasks this difference is presumably due to the increase in response information. This increase in CUD was accompanied by reduced accuracy in the crossed conditions, suggesting difficulty in transferring the extra response information. L.B.'s accuracy in the crossed conditions dropped even further in the three-choice task. However there was again a directional difference: L.B.'s left hand-RVF responses were less accurate $(75 \%$ in the two-choice and $58 \%$ in the three-choice task) than right hand-LVF responses ( 83 and $81 \%$, respectively). This was also apparent in the amount of information transferred -0.21 and 0.27 bits in the two- and threechoice task compared to 0.47 and 0.95 bits, respectively. This implies that transfer from the right to the left hemisphere is better than that from left to right, and that the left hemisphere may be superior in deciphering transferred information. Even in the right hand-LVF combination, however, the amount of information transferred was still less than 1 bit.

\section{General discussion}

\subsection{Normal subjects}

In both experiments, RT increased with increasing task complexity, reflecting an increase in processing demands and greater cerebral involvement. However this was not matched by an increase in CUD, which actually tended to diminish. Indeed, the CUD was consistently negative for the two-choice and threechoice tasks, and significantly so in the case of the three-choice task in Experiment 2, suggesting that performance might be actually enhanced under the crossed condition. This enhancement might be attributed to shared processing, with one hemisphere carrying out the stimulus discrimination and the other the response selection, allowing a degree of parallel processing.

There is other evidence that performance can be more efficient when information is directed to both hemispheres than when it is directed to only one. This may depend, however, on the complexity of the task.
For example, when subjects are required to decide whether two letters are physically identical (e.g., A and A), responses are faster if the letters are presented in the same visual field than if they are presented in opposite visual fields, but when they are required to decide whether the letters have the same name (e.g., A and a) responses are faster when they are presented to opposite fields [3]. Reviewing these and other findings, Banich [2] suggests that there is a trade off between the disadvantage of interhemispheric transfer and the advantage of parallel processing in the two hemispheres. As the complexity of the task increases, so the advantage of parallel processing increases, and performance becomes more efficient when information is shared between the hemispheres than when it is directed to a single hemisphere.

However, the studies reviewed by Banich [2] do not have immediate application to the present experiments, since they have to do with the allocation of stimulus processing. In the present study, any comparable advantage accruing to the crossed conditions would imply that stimulus information is processed in one hemisphere and response processing is carried out in the other. But some experiments imply that stimulus information is presented to one hemisphere while the response processing is carried out in the other. Since the responses are contingent on the stimuli, this means that parallel processing can be only partial, at best, with perhaps some overlap between stimulus processing and the preparation of response, as in the cascade model of sequential processing [35]. Even so, the data are consistent with Banich's suggestion that the advantages of sharing processing between hemispheres are more pronounced in complex than in simple tasks.

\subsection{The acallosal subject R.B.}

The main conclusion to be drawn from R.B.'s results is that he had little difficult with any of the tasks in the crossed condition; in terms of both accuracy and RT his results resembled those of the normals rather than those of the commissurotomised subject L.B. This confirms previous evidence that callosal agenesis does not simply mimic commissurotomy in its effects $[29,30]$. Precisely how R.B. (and other callosal agenics) compensate for the absence of the corpus callosum remains unclear. Perhaps the most likely channel of interhemispheric transfer of colour and form is the anterior commissure, although it is also possible that control of response in the experiments reported here was accomplished via ipsilateral pathways under the crossed conditions.

R.B.'s CUDs were too variable for clear interpretation, perhaps because there were too few trials for stable RT estimates. However, except in the case of the three-choice colour discrimination, his CUDs were 
always below the estimate of $23-25$ ms derived from our previous studies using simple RT [13,21], and always within the range of values in the normal sample. This suggests that, as in the normal subjects, the CUD tends to decrease, if anything, as the complexity of the task increases.

\subsection{The commissurotomised subject L.B.}

In contrast to R.B., the commissurotomized subject L.B.'s CUD was about the same in the go-no go task as in simple RT tasks [13,21], and increased with task complexity. However, the CUDs for the two-choice and, more especially, the three-choice task are suspect, since L.B.'s accuracy dropped dramatically with increasing complexity. L.B. clearly had difficulty transferring information interhemispherically, especially in the crossed conditions of the three-choice task. Previous evidence has shown L.B. to be impaired in tasks requiring integration of information about colour or shape, but that he is nevertheless sometimes capable of performance at better than chance $[38,44]$. It has been pointed out that the level of performance he actually achieves can often be explained by the transfer of mere binary information (i.e., 1 bit) $[11,14,17]$. This might even be accomplished by subtle cross-cueing; for example, he might be able to signal whether a colour is red or not by raising or lowering the tongue. Informational analyses in the present study show that the amount of information transmitted in the crossed conditions never in fact exceeded one bit. Moreover, it appears that the CUD was dependent on the amount of response information, not the amount of stimulus information, since it was longer for the two-choice task than for the go-no go task. This adds to the evidence that response information, not stimulus information, is transferred [10,21].

An interesting aspect of L.B.'s performance is that he showed a left-hand advantage in the go-no go tasks, as he had in simple RT [21], but a left visualfield advantage in the two-choice tasks. Both effects are probably attributable to some left-hemispheric dysfunction, of recent origin. What is not clear is why this dysfunction should result in slowed response execution in simple tasks, but slowed stimulus processing in more complex discrimination tasks.

It was also clear that L.B.'s ability to transmit information between hemispheres was asymmetrical. His accuracy in the right hand-LVF conditions was well above that in the left hand-RVF conditions, both in terms of matching responses to colours or shapes and in terms of overall accuracy. Since L.B. is righthanded, it may well be the case that there is more sophisticated transfer of response information from right to left hemisphere than vice versa, since the left hemisphere appears to be dominant for response selec- tion [41]. Marzi et al. [34] have shown similarly that there is more rapid transfer from right to left hemisphere than from left to right in simple RT in righthanded normals. The present results may also imply that the left hemisphere is superior to the right in deciphering coarse transferred information. It is also possible, however, that this asymmetry is related to L.B.'s left-hemisphere pathology, resulting in a deficit of transfer from the left hemisphere to the right.

But although transfer was more effective from right hemisphere to left, it appeared that cross-cueing was more likely from the left hemisphere to the right, at least in the transfer of colour information. In Experiment 1, in the three-choice task, L.B. was observed to be staring at his hand before making the response. However, when he was prevented from doing so, his performance accuracy dropped only when lefthand responses were required to RVF stimuli, suggesting that it was the left hemisphere that initiated the strategy.

\subsection{Summary}

In summary, the present data support Poffenberger's conjecture that the CUD is not a valid measure of IHTT in tasks more complex than simple RT. As the processing requirements increase, so the advantages of shared processing between the hemispheres increase; crossed conditions may then allow a degree of overlap in the processing of stimulus and response, resulting in a shortening of RT that may cancel the time lost through interhemispheric transfer, and even result in RTs that are shorter than those under the uncrossed condition. For the most part, the acallosal subject R.B. appeared also to profit from shared processing, although there was also some evidence for prolonged interhemispheric transfer. In contrast, the commissurotomized subject L.B. showed clear evidence of disconnection, with poor interhemispheric transfer in the discrimination of both colour and shape. The results also give quantitative support to previous conjectures that interhemispheric transfer in the case of L.B. does not exceed 1 bit (binary digit) of information.

\section{Acknowledgements}

This research was supported in part by a grant from the Marsden Fund of New Zealand, and in part by grants from the Auckland University Research Committee. We thank Salvatore Aglioti, Dahlia Zaidel, and Eran Zaidel for help in contacting and testing the patients. We also thank L.B. and R.B. for their willing cooperation. 


\section{References}

[1] Aglioti S, Berlucchi G, Pallini R, Rossi G, Tassinari G. Hemispheric control of unilateral and bilateral responses to lateralized light stimuli after callosotomy and in callosal agenesis. Experimental Brain Research 1993;95:151-65.

[2] Banich MT. Interhemispheric processing: theoretical considerations and empirical approaches. In: Davidson R, Hugdahl K, editors. Brain asymmetry. Cambridge, MA: MIT Press, 1995. p. 427-50.

[3] Banich MT, Belger A. Interhemispheric interaction: how do the hemispheres divide and conquer a task? Cortex 1990;26:77-94.

[4] Bashore TR. Vocal and manual reaction time estimates of interhemispheric transmission time. Psychological Bulletin 1981;89:352-68.

[5] Berlucchi G, Aglioti S, Marzi CA, Tassinari G. Corpus callosum and simple visuomotor integration. Neuropsychologia 1995;33:923-36.

[6] Bogen JE, Schultze DH, Vogel PJ. Completeness of callosotomy shown by magnetic resonance imaging in the long term. Archives of Neurology 1988;45:1203-5.

[7] Bogen JE, Vogel PJ. Neurologic status in the long term following complete cerebral commissurotomy. In: Michel F, Schott B, editors. Les syndromes de disconnexion calleuse chez l'homme. Lyon: Hopital Neurologique, 1975. p. 227-51.

[8] Braun CMJ. Estimation of interhemispheric dynamics from simple unimanual reaction to extrafoveal stimuli. Neuropsychology Review 1992;3:321-65.

[9] Brysbaert M. Behavioural estimates of interhemispheric transmission time and the signal detection method: a reappraisal. Perception and Psychophysics 1994;56:479-90.

[10] Clarke JM, Zaidel E. Simple reaction times to lateralized light flashes. Brain 1989;112:849-70.

[11] Corballis MC. Visual integration in the split-brain. Neuropsychologia 1995;33:937-59.

[12] Corballis MC. A dissociation in naming digits and colours following commissurotomy. Cortex 1996;32:515-25.

[13] Corballis MC. Interhemispheric neural summation in the absence of the corpus callosum. Brain 1998;121:1795-807.

[14] Corballis MC. Can commissurotomized subjects compare digits between the visual fields? Neuropsychologia 1994;32:1475-86.

[15] Corballis MC, Boyd L, Schulze A, Rutherford B. Role of the commissures in interhemispheric temporal judgments. Neuropsychology 1998;12:519-25.

[16] Corballis MC, Sergent J. Imagery in a commissurotomized patient. Neuropsychologia 1988;26:13-26.

[17] Corballis MC, Sergent J. Judgements of numerosity by commissurotomized subjects. Neuropsychologia 1992;30:865-76.

[18] Corballis MC, Trudel CI. Role of the forebrain commissures in interhemispheric integration. Neuropsychology 1993;7:306-24.

[19] Fendrich R, Gazzanaiga MS. Evidence of foveal splitting in a commissurotomy patient. Neuropsychologia 1989;27:273-81.

[20] Fiorentini A, Berardi N, Falsini B, Porciatti V. Interhemispheric transfer of visual perceptual learning in callosal agenesis. Clinical Vision Science 1992;7:133-4.

[21] Forster B, Corballis MC. Interhemispheric transmission times in the presence and absence of the forebrain commissures: Effects of luminance and equiluminance. Neuropsychologia 1998;36:925-34.

[22] Garner WR, Hake HW. The amount of information in absolute judgements. Psychological Review 1951;58:446-59.

[23] Holtzman JD. Interactions between cortical and subcortical visual areas: evidence from human commissurotomy patients. Vision Research 1984;24:801-13.

[24] Jeeves MA. A comparison of interhemispheric transmission times in acallosals and normals. Psychonomic Science 1969;16:245-6.

[25] Jeeves MA. Callosal agenesis - a natural split-brain overview. In: Lassonde M, Jeeves MA, editors. Callosal agenesis. New York: Plenum Press, 1994. p. 285-99.

[26] Johnson LE. Bilateral visual cross-integration by human forebrain commissurotomized subjects. Neuropsychologia 1984;22:167-75.

[27] Karnath HO, Schumacher M, Wallesch CW. Limitations of interhemispheric extracallosal transfer of visual information in callosal agenesis. Cortex 1991;27:345-50.

[28] Kinsbourne M, Fisher M. Latency of uncrosses and crossed reaction in callosal agenesis. Neuropsychologia 1971;9:471-3.

[29] Lassonde M. Disconnection syndrome in callosal agenesis. In: Lassonde M, Jeeves MA, editors. Callosal agenesis. New York: Plenum Press, 1994. p. 275-84.

[30] Lassonde M, Sauerwein HC, Chicoine A-J, Geoffroy G. Absence of the disconnection syndrome in callosal agenesis and early callosotomy: brain reorganization or lack of structural specificity during ontogeny? Neuropsychologia 1991;29:481-95.

[31] Lassonde M, Sauerwein HC, Lepore F. Extent and limitations of callosal plasticity: presence of disconnection symptoms in callosal agenesis. Neuropsychologia 1995;33:989-1007.

[32] Lian A, Bjørklund RA. Crossed-uncrossed difference (CUD) on reaction time and movement time in three two-choice experiments. Scandinavian Journal of Psychology 1995;36:208-24.

[33] Martin A. A qualitative limitation on visual transfer via the anterior commissure: evidence from a case of callosal agenesis. Brain 1985;108:43-63.

[34] Marzi CA, Bisiacchi P, Nicoletti R. Is interhemispheric transfer of visuomotor information asymmetric? Evidence from a metaanalysis. Neuropsychologia 1991;29:1163-77.

[35] McClelland JL. On the time relations of mental processes: an examination of systems of processing in cascade. Psychological Review 1979;86:287-330.

[36] Milner AD, Jeeves MA. A review of behavioural studies of agenesis of the corpus callosum. In: Russell IS, van Hof MW, Berlucchi GM, editors. Structure and function of the cerebral commissures. London: Macmillan, 1979.

[37] Milner AD, Jeeves MA, Silver PH, Lines CR, Wilson J. Reaction times to lateralized visual stimuli in callosal agenesis: stimulus and response factors. Neuropsychologia 1985;23:32331.

[38] Myers JJ, Sperry RW. Interhemispheric communication after section of the forebrain commissures. Cortex 1985;21:249-60.

[39] Poffenberger AT. Reaction time to retinal stimulation with special reference to the time lost in conduction through nerve centres. Archives of Psychology 1912;23:1-73.

[40] Reuter-Lorenz PA, Nozawa G, Gazzaniger MS, Hughes HC. Gate of neglected targets: a chronometric analysis of redundant target effects in the bisected brain. Journal of Experimental Psychology: Human Perception and Performance 1995;21:21130.

[41] Rushworth MFS, Nixon PD, Wade DT, Renowden S, Passingham RE. The left hemisphere and the selection of learned actions. Neuropsychologia 1998;36:11-24.

[42] Sauerwein H, Lassonde MC. Intra- and interhemispheric processing of visual information in callosal agenesis. Neuropsychologia 1983;21:167-71.

[43] Schneider W Micro Experimental Laboratory. Psychological Software Tools, Pittsburgh, PA 1995.

[44] Sergent J. Subcortical coordination of hemisphere activity in commissrotmized patients. Brain 1986;109:357-69.

[45] Sergent J, Myers JJ. Manual, blowing, and verbal simple reactions to lateralized flashes of light in commissurotomized patients. Perception and Psychophysics 1985;37:571-8.

[46] Tassinari G, Aglioti S, Pallini R, Berlucchi G, Rossi GF. 
Interhemispheric integration of simple visuomotor responses in patients with partial callosal defects. Behavioural Brain Research 1994;64:141-9.

[47] Trevarthen C, Sperry RW. Perceptual unity of ambient visual field in human commissurotomy patients. Brain 1973;96:547-70.
[48] Umiltá C, Frost N, Hyman R. Interhemispheric effects on choice reaction times to one-, two-, and three-letter displays. Journal of Experimental Psychology 1972;93:198-204.

[49] Winer BJ. Statistical principles in experimental design. New York: Wiley, 1971. 